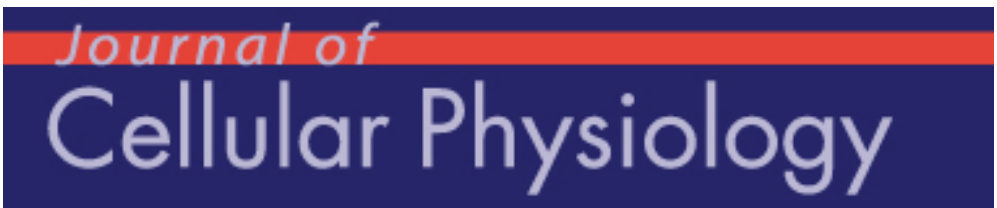

\title{
DIFFERENTIAL STRUCTURAL FEATURES IN SOLEUS AND GASTROCNEMIUS OF CARNITINE-TREATED CANCER CACHECTIC RATS
}

\begin{tabular}{|c|c|}
\hline Journal: & Journal of Cellular Physiology \\
\hline Manuscript ID & JCP-18-2602.R2 \\
\hline Wiley - Manuscript type: & Original Research Article \\
\hline $\begin{array}{r}\text { Date Submitted by the } \\
\text { Author: }\end{array}$ & $\mathrm{n} / \mathrm{a}$ \\
\hline Complete List of Authors: & $\begin{array}{l}\text { Salazar-Degracia, Anna; IMIM, PRBB, URMAR } \\
\text { Busquets, Silvia; Universitat de Barcelona, Barcelona, and Institut de } \\
\text { Biomedicina de la Universitat de Barcelona (IBUB), Cancer Research } \\
\text { Group, Departament de Bioquímica i Biomedicina Molecular, Facultat de } \\
\text { Biologia } \\
\text { Argiles, Josep M; Universitat de Barcelona, Barcelona, and Institut de } \\
\text { Biomedicina de la Universitat de Barcelona (IBUB) } \\
\text { Serpe, Roberto; University of Cagliari, Department of Medical Sciences } \\
\text { and Public Health "M. Aresu" } \\
\text { Perez-Peiro, Maria; IMIM-Hospital del Mar, Parc de Salut Mar, Health and } \\
\text { Experimental Sciences Department (CEXS), Universitat Pompeu Fabra } \\
\text { (UPF), Pulmonology Department-Muscle Wasting and Cachexia in Chronic } \\
\text { Respiratory Diseases and Lung Cancer Research Group } \\
\text { Rojano-Toimil, Alba; IMIM-Hospital del Mar, UPF, PRBB, CIBERES, } \\
\text { Pulmonology-URMAR } \\
\text { Lopez-Soriano, Francisco J; Universitat de Barcelona, Barcelona, and } \\
\text { Institut de Biomedicina de la Universitat de Barcelona (IBUB) } \\
\text { Barreiro, Esther; IMIM-Hospital del Mar, UPF, PRBB, CIBERES, } \\
\text { Pulmonology-URMAR }\end{array}$ \\
\hline Key Words: & $\begin{array}{l}\text { experimental cancer-induced cachexia, slow- and fast-twitch muscles, L- } \\
\text { carnitine, muscle fiber type and morphometry, muscle structure and } \\
\text { morphology }\end{array}$ \\
\hline
\end{tabular}

\section{SCHOLARONE ${ }^{m}$ Manuscripts}


DIFFERENTIAL STRUCTURAL FEATURES IN SOLEUS AND GASTROCNEMIUS

Anna Salazar-Degracia ${ }^{1}$, Sílvia Busquets ${ }^{2}$, Josep M. Argilés ${ }^{2}$, Roberto Serpe ${ }^{3}$, Maria Pérez-

Peiró$^{1}$, Alba Rojano-Toimil ${ }^{1}$, Francisco J. López-Soriano², and Esther Barreiro ${ }^{1}$ 
26 Spanish Ministry of Science and Innovation, contract grant number SAF 2011-26091, 27 Spanish Respiratory Society (SEPAR), contract grant numbers, SEPAR 2016, SEPAR 2018, 28 Catalan Foundation of Pulmonology (FUCAP), contract grant numbers, FUCAP 2016. 


\section{ABSTRACT}

Muscle wasting is associated with chronic diseases and cancer. Elucidation of the biological mechanism involved in the process of muscle mass loss and cachexia may help identify therapeutic targets. We hypothesized that L-carnitine treatment may differentially revert muscle fiber atrophy and other structural alterations in slow- and fast-twitch limb muscles of rats bearing the Yoshida ascites hepatoma. In soleus and gastrocnemius of tumor-bearing rats (10 $\mathrm{AH}-130$ Yoshida ascites hepatoma cells inoculated intraperitoneally) with and without treatment with L-carnitine ( $1 \mathrm{~g} / \mathrm{kg}$ body weight for seven days, intragastric), food intake, body and muscle weights, fiber typing and morphometry, morphological features, redox balance, autophagy and proteolytic and signaling markers were explored. Levels of carnitine palmitoyl transferase were also measured in all the study muscles. L-carnitine treatment ameliorated the atrophy of both slow- and fast-twitch fibers (gastrocnemius particularly), muscle structural alterations (both muscles), and attenuated oxidative stress, proteolytic and signaling markers (gastrocnemius). Despite that carnitine palmitoyltransferase-1 levels increased in both muscle types in a similar fashion, L-carnitine ameliorated muscle atrophy and proteolysis in a muscle-specific manner in cancer-induced cachexia. These data reveal the need to study muscles of different fiber type composition and function to better understand whereby Lcarnitine exerts its beneficial effects on the myofibers in muscle wasting processes. These findings also have potential clinical implications, since combinations of various exercise and muscle training modalities with L-carnitine should be specifically targeted for the muscle groups to be trained.

\section{Word count: 235}

KEY WORDS: experimental cancer-induced cachexia; slow- and fast-twitch muscles; Lcarnitine; muscle fiber type and morphometry; muscle structure and morphology; proteolytic, autophagy, and signaling markers 


\section{INTRODUCTION}

Loss of muscle mass and malnutrition are associated with chronic respiratory and cardiac conditions and cancer (Barreiro et al, 2015;Barreiro, 2017;Fearon et al, 2011;Cederholm et al, 2019). Cachexia defined as the process of body weight loss of more than $5 \%$ over one year or shorter in the context of a chronic illness (e.g. renal failure, cancer, chronic respiratory and cardiac diseases) is a devastating condition that is associated with greater mortality irrespective of the primary disorder (Barreiro et al, 2015;Barreiro, 2017;Fearon et al, 2011;Alvarez et al, 2016;Gonzalez and de-Torres, 2017;Izquierdo Alonso, 2016; von and Anker, 2014).

Several etiologic factors and biological mechanisms have been described as part of the pathophysiology of body and muscle mass loss in patients with chronic conditions including cancer (Barreiro et al, 2015;Barreiro, 2017;Busquets et al, 2004;Fearon et al, 2011;Salazar-Degracia et al, 2016; Toledo et al, 2011;Toledo et al, 2014; Toledo et al, 2016). As such increased oxidative stress, systemic inflammation, metabolic derangements, increased proteolysis, poor anabolism, epigenetic modifications, specific atrophy signaling pathways, and alterations in muscle morphology have been demonstrated to occur in the skeletal muscle fibers of patients with muscle wasting and in experimental models (Barreiro et al, 2015;Barreiro, 2017;Busquets et al, 2004;Fearon et al, 2011;Puig-Vilanova et al, 2014;Puig-Vilanova et al, 2015;Salazar-Degracia et al, 2016;Salazar-Degracia et al, 2017; Toledo et al, 2011; Toledo et al, 2014; Toledo et al, 2016).

Elucidation of the biological mechanism involved in the process of muscle mass loss and cachexia is of paramount importance as they may help identify therapeutic targets. In this respect, several treatments have proven to attenuate or even prevent muscle mass loss through several biological mechanisms, particularly in experimental models of cancer cachexia (Busquets et al, 2004;Busquets et al, 2011;Busquets et al, 2012b;Busquets et al, 2012a;Carter 
et al, 2004;Fearon et al, 2011;Laviano et al, 2011;Puig-Vilanova et al, 2014;Puig-Vilanova et al, 2015;Salazar-Degracia et al, 2017;Silverio et al, 2011;Toledo et al, 2014;Toledo et al, 2016). In this regard, growth hormone analogues, anabolic steroids, beta 2 -agonists (e.g. formoterol), and appetite stimulants have been proposed as potential therapeutic strategies for the treatment of cachexia associated with cancer and other chronic conditions (Fearon et al, 2011; Laviano et al, 2011;Silverio et al, 2011;von and Anker, 2014).

In patients with advanced pancreatic cancer (Kraft et al, 2012), oral treatment with L-carnitine induced a significant improvement in body weight and fat body compartment. Another study also revealed that L-carnitine concentration may be used as a marker to evaluate muscle mass loss in the cachexia associated with cancer in patients (Szefel et al, 2012). In mice bearing colon cancer cells, L-carnitine significantly improved body, muscle, and fat weight and nutritional parameters through the upregulation of carnitine palmitoyl transferase (Liu et al, 2011). Importantly, among several clinical parameters, mean body lean mass significantly increased along with the quality of life in patients with severe cancer cachexia in response to treatment with L-carnitine for several weeks (Gramignano et al, 2006). In keeping with, it was also demonstrated that treatment with L-carnitine in rats bearing the AH-130 Yoshida ascites hepatoma ameliorated body and muscle weights along with physical activity probably through reduced proteasome activity (Busquets et al, 2012a). Whether the expression of several biological events leading to muscle wasting in response to L-carnitine may differ in slow- and fast-twitch muscle fibers in models of cancer-induced cachexia need to be elucidated. Moreover, the effects of L-carnitine on muscle phenotype and structure in slow- and fast-twitch muscles also remains an open question.

On this basis, we hypothesized that L-carnitine treatment may differentially revert muscle fiber atrophy and other muscle morphological features in slow- and fast-twitch limb muscles of rats bearing the Yoshida ascites hepatoma, a well-validated model of experimental 
cancer-induced cachexia (Busquets et al, 2004;Busquets et al, 2011;Busquets et al, 2012b;Busquets et al, 2012a;Salazar-Degracia et al, 2017;Toledo et al, 2011;Toledo et al, 2014; Toledo et al, 2016). Accordingly, we sought to investigate in gastrocnemius and soleus of cancer-induced cachectic rats that were treated with L-carnitine the following markers: 1) total body, muscle, and tumor weights, 2) levels of muscle morphological features, 3) muscle fiber type and morphometry, and 4) markers of proteolysis, autophagy and signaling pathways known to be involved in protein breakdown and metabolism. A control group of non-treated rats bearing the ascites hepatoma tumor was also used for the purpose of the investigation. Protein levels of the enzyme carnitine palmitoyl transferase-1 were also assessed as a marker of carnitine effects in both muscle types of all study animals.

\section{MATERIALS AND METHODS}

(See detailed methodologies in the online supplementary material)

\section{Data will be available upon request to the Authors.}

\section{Animal experiments and design}

Male Wistar rats $(\sim 125$ grams, 5 weeks of age $)$ were purchased from Harlan Interfauna Ibérica SL (Barcelona, Spain). Rats were kept under pathogen-free conditions in individual cages and maintained at a constant temperature $22 \pm 2{ }^{\circ} \mathrm{C}$ with a regular $12: 12$ hour light-dark cycle in the animal house facilities at Universitat de Barcelona.

In the rats, cachexia was induced as a result of an intraperitoneal injection of $10^{8} \mathrm{AH}-130$ Yoshida ascites hepatoma cells, a well-validated model of cancer-induced cachexia, obtained from exponential tumors as reported in previous studies by our group (Busquets et al, 2004;Busquets et al, 2011;Busquets et al, 2012b;Busquets et al, 2012a;Salazar-Degracia et al, 2017; Toledo et al, 2011;Toledo et al, 2014;Toledo et al, 2016). The loss of body weight and muscle mass is progressive and fast in the rats. Importantly, moderate cachexia $(8 \%$ of body 
weight loss) was already seen on day 4 , and on day 7 , the rats experienced $20-25 \%$ of body weight loss as was also reported in previous investigations (Lopez-Soriano et al, 1997;Toledo et al, 2016). For ethical reasons, seven days was established as the duration of the study, the day at which large tumor sizes were reached. Rats were randomly divided into two groups ( $\mathrm{N}=8$ /group) as follows: 1) cancer-cachexia and 2) cancer-cachexia treated with L-carnitine. L-carnitine was administered intragastrically in the rats $(1 \mathrm{~g} / \mathrm{kg}$ body weight, Sigma-Tau, Barcelona, Spain) 6 hours following inoculation of the tumor cells. Thereafter, the same dose of L-carnitine was administered every 24 hours for seven consecutive days up until the sacrifice of the animals on day seven. Non-treated rats received the corresponding volume of solvent (corn oil) that was also administered intragastrically every 24 hours for seven days up until the day of animal sacrifice (Busquets et al, 2012a).

\section{Ethics}

All animal experiments were conducted in the animal facilities at Facultat de Biologia, Universitat de Barcelona (Barcelona). This controlled study was designed in accordance with both the ethical standards on animal experimentation in our institution (EU 2010/63 CEE and Real Decreto 53/2013 BOE 34, Spain) and the Helsinki convention for the use and care of animals. All experiments were approved by the Institutional Animal Research Committee (Universitat de Barcelona).

\section{In vivo measurements in the animals}

Food and water were supplied ad libitum for the entire duration of the study period. In all animal groups, food intake and body weight were determined on day 0 and immediately prior to their sacrifice on day 7. Food intake was calculated as follows: (food weight on day 7 food weight on day 0 ) / initial body weight on day $0 \times 100$. Tumor weights were determined in all the rats during their sacrifice. The following equation was used to estimate the percentage of body weight gain at the end of the study period in both groups of rats: [(body 
weight on day 7 - tumor weight on day 7) - body weight on day 0$] /$ body weight on day $0 \mathrm{x}$ 100 (Busquets et al, 2012b;Busquets et al, 2012a;Salazar-Degracia et al, 2017;Toledo et al, 2011;Toledo et al, 2014;Toledo et al, 2016;Busquets et al, 2012b).

\section{Sample collection}

As abovementioned rats in both study groups were sacrificed on day seven following tumor inoculation. Body weights were estimated using a specific scale right before the animal sacrifice. Rats were anesthetized as a result of an intraperitoneal injection of $3: 1$ ketamine/xylazine mixture (Imalgene 1000, Rhone Merieux, France and Rompun ${ }^{\circledR}$ and Bayer AG, Leverkusen, Germany, respectively). In all the rats, total anesthetic depth was confirmed by evaluating the pedal and blink reflexes. The gastrocnemius and soleus muscles were carefully dissected and obtained in full for the purpose of the study. In all rats, one piece of the muscle specimen was snap-frozen in liquid nitrogen to be stored at $-80^{\circ} \mathrm{C}$ for molecular analyses, while the second fragment was paraffin-embedded for the analyses of fiber phenotype (composition and morphometry) and the quantification of specific muscle morphological features, respectively (Busquets et al, 2012b;Busquets et al, 2012a;SalazarDegracia et al, 2017;Toledo et al, 2011;Toledo et al, 2014;Toledo et al, 2016).

\section{Muscle biology analyses}

All the muscle biological experiments were performed in the same laboratory at Hospital del Mar-IMIM-Universitat Pompeu Fabra (Barcelona).

\section{Muscle fiber counts and morphometry}

In both study groups of rats, MyHC-I and -II isoforms were identified using anti-MyHC-II antibody (Sigma-Aldrich, St. Louis, MO, USA) on three-micrometer muscle paraffinembedded sections from the gastrocnemius and soleus muscles as previously described in studies from our group (Chacon-Cabrera et al, 2014;Chacon-Cabrera et al, 2015;ChaconCabrera et al, 2016b;Chacon-Cabrera et al, 2016a;Chacon-Cabrera et al, 2017;Fermoselle et 
al, 2012;Salazar-Degracia et al, 2016). Brown stained myofibers were those that positively reacted with anti-MyHC type II antibody, whereas type I fibers were not stained (white color).

\section{Muscle morphological features}

In the gastrocnemius and soleus muscles of both groups of rats, the area fraction of normal and abnormal muscle was assessed on three-micrometer paraffin-embedded sections as also previously described by our group (Chacon-Cabrera et al, 2014; Chacon-Cabrera et al, 2016b;Puig-Vilanova et al, 2014;Salazar-Degracia et al, 2016). Muscle sections were stained with hematoxylin-eosin. In order to quantify the proportion of muscle morphological features in the study muscles, quantitative analyses were conducted using computer-assisted morphometric techniques in all the sections as previously reported (Chacon-Cabrera et al, 2014;Chacon-Cabrera et al, 2016b;Puig-Vilanova et al, 2014;Salazar-Degracia et al, 2016).

\section{Immunoblotting}

Immunoblotting techniques were employed in order to explore protein levels of the different antigens and molecular markers determined in the investigation following standard procedures and previous studies (Chacon-Cabrera et al, 2014;Chacon-Cabrera et al, 2016b;Chacon-Cabrera et al, 2016a;Chacon-Cabrera et al, 2017;Puig-Vilanova et al, 2014;Puig-Vilanova et al, 2015;Salazar-Degracia et al, 2016;Salazar-Degracia et al, 2017; Chacon-Cabrera et al, 2015;Fermoselle et al, 2012).

Protein levels were identified in the gastrocnemius and soleus muscles using specific antibodies described as follows: anti-carnitine palmitoyl transferase-1 (CPT1A, 1:1000, ab128568) antibody from Abcam (Cambridge, UK), anti-malondialdehyde (MDA; 1:4000, MD20A-G1b) antibody from Academy Bio-Medical Company (Houston, TX, USA), anticatalase $(1: 2000,219010)$ antibody from Merk Millipore (Burlington, MA, USA), anti-total protein ubiquitination (1:5000, A-100) antibody from Boston Biochem (Cambridge, MA, USA), anti-mammalian target of rapamycin (mTOR; 1:1000, \#2972S), anti-serine/threonine 
206 kinase 1 (AKT; 1:1000, \#9272S), anti-microtubule-associated protein 1 light chain 3B

207

208

(LC3B; 1:1000, \#2775S) antibodies from Cell signaling, anti-nucleoporin p62 (p62; 1:1000, P0067) antibody from Sigma-Aldrich (St. Louis, MO, USA), anti-transcription factors forkhead box O-3 (FoxO3, 1:500, AP20683PU-N) antibody from Acris (Aachen, Germany), antiNAD-dependent protein deacetylase sirtuin-1 (Sirtuin-1, 1:1000, 13161-1-AP) antibody from ProteinTech (Manchester, UK), anti-superoxide dismutase 2 (SOD2, 1:5000, sc-30080), antiSOD1 (1:2000, sc-11407), anti-muscle ring finger protein-1 (MuRF-1; 1:2000, sc-27642), anti-atrogin-1 (1:1000, sc-166806), anti-peroxisome proliferator-activated receptor gamma coactivator (PGC) 1-alpha (1:500, sc-13067), anti-nuclear factor kappa-light-chain-enhancer of activated B cells (NF-kB) p65 (1:500, sc-8008), and anti-glyceraldehyde 3-phosphate dehydrogenase (GAPDH; 1:2000, sc-25778) antibodies from Santa Cruz (Santa Cruz, CA, USA).

Importantly, in order to detect expression levels of the loading control GAPDH, standard stripping methodologies were used independently for each of the antigens analyzed in the investigation: CPT1A, MDA, SOD1, SOD2, Catalase, FoxO-3, Sirtuin-1, PGC-1alpha, NFkB p65, AKT, mTOR, protein ubiquitin, atrogin-1, MuRF-1, p62 and LC3B.

\section{Statistical Analysis}

In the study, the variables are represented as a mean and standard deviation in both tables and graphs. The normality of the study variables was checked using the Shapiro-Wilk test. The Student's T-test was used to assess potential significant differences between the two study groups of rats (cancer-cachexia and cancer-cachexia + carnitine) in each muscle independently (gastrocnemius and soleus). A level of significance of $p \leq 0.05$ was established and applied to all analyses. The sample size chosen was partly based on previous investigations (Busquets et al, 2004;Busquets et al, 2011;Busquets et al, 2012b;Busquets et al, 2012a;Chacon-Cabrera et al, 2014; Chacon-Cabrera et al, 2015; Chacon-Cabrera et al, 
231 2016b;Chacon-Cabrera et al, 2016a;Chacon-Cabrera et al, 2017;Salazar-Degracia et al, 2016;Salazar-Degracia et al, 2017;Toledo et al, 2011;Toledo et al, 2014;Toledo et al, 2016) outcomes. The Statistical Package for the Social Science (Portable SPSS, PASW statistics 18.0 version for Windows, SPSS Inc., Chicago, IL, USA) was used to conduct all the statistical analyses in the study.

\section{RESULTS}

\section{Physiological characteristics of the study animals}

At the end of the study period, food intake was greater in the cachectic rats treated with Lcarnitine than in non-treated cachectic rodents (Table 1). In the cachectic rats treated with Lcarnitine, the parameters body weight gain and gastrocnemius and soleus muscle weights significantly improved compared to non-treated cancer-cachexia animals (Table 1). Protein levels of carnitine palmitoyl transferase-1 enzyme were higher in gastrocnemius and soleus muscles of cancer-cachexia rats treated with L-carnitine compared to the non-treated cachectic animals (Figure 1).

\section{Effects of L-carnitine on muscle phenotype and structure}

The proportions of slow- and fast-twitch muscle fibers did not significantly differ between the two study groups (Table 2 and Figure 2). In cachectic rats treated with L-carnitine, the size of both slow- and fast-twitch fibers was significantly greater in the gastrocnemius muscle, while in the soleus only the size of the slow-twitch fibers significantly improved compared to nontreated cachectic animals (Table 2 and Figure 2). Total muscle morphological features and internal nuclei counts significantly decreased in both types of muscles: gastrocnemius and soleus of cancer-cachexia rats treated with L-carnitine compared to non-treated cachectic animals (Figures 3A-3D).

\section{Effects of L-carnitine on redox balance in muscles}


In the gastrocnemius muscle, levels of the protein oxidation marker MDA-protein adducts were significantly lower in the cancer-cachexia rats treated with L-carnitine compared to the non-treated cachectic rats (Figures 4A-4B), while no differences were seen in the soleus muscle (Figures 4C-4D). Protein levels of the antioxidants SOD1, SOD2, and catalase did not significantly differ between the study groups in any muscle type (Figures 4A-4D).

\section{Effects of L-carnitine on atrophy signaling pathways in muscles}

In the gastrocnemius muscle, treatment with L-carnitine did not modify the protein levels of Sirtuin-1, PGC-1 alpha and NF-kB p65, whereas a significant decline in FoxO-3 protein content was seen in the cancer-cachexia rats (Figures 5A-B). In the soleus, no differences were observed in the levels of atrophy signaling markers between cancer-cachexia rats treated with L-carnitine and the non-treated animals (Figures 5C-D). Protein levels of Akt and mTOR did not significantly differ between the two study groups in any muscle type (Figures 6A-D).

\section{Effects of L-carnitine on proteolytic and autophagy markers in muscles}

Compared to cancer-cachexia rats, levels of total protein ubiquitination, MuRF-1, and atrogin1 significantly decreased in the gastrocnemius muscle of cancer-cachexia rats treated with Lcarnitine, whereas no significant differences were seen in the soleus for any of these markers (Figures 7A-D). Treatment of the cachectic rats with L-carnitine did not elicit any significant effect on protein levels of the autophagy markers p62 and LC3B in either the gastrocnemius or soleus muscles (Figures (8A-8D).

\section{DISCUSSION}

In the current study, despite that levels of carnitine palmitoyl transferase significantly increased in both muscle types of the treated animals in a similar fashion, differential phenotypic and proteolytic features were observed between the slow-twitch soleus and the fast-twitch gastrocnemius. Specifically, in cancer cachectic rats treated with L-carnitine for 
281 the entire duration of the experimental period (seven days), body and muscle (both slow- and

282 fast-twitch muscle types) weights significantly recovered along with the significant decline in muscle morphological features detected in both limb muscle types. In the gastrocnemius, the degree of muscle atrophy of type I and type II fibers significantly ameliorated, whereas only the size of the slow-twitch fibers recovered in the soleus of the L-carnitine-treated rats. In the latter muscle, no significant improvements in protein expression of proteolytic or signaling markers were observed. L-carnitine therapy did not elicit any significant effects on the tumor weights of the treated cachectic rats. The relevance of the study findings is discussed below.

Carnitine is a permanently charged ammonium cation that carries long-chain activated fatty acids from the cytoplasm into the mitochondrial matrix to be processed by oxidation to produce adenosine triphosphate (ATP). Carnitine, which is formed from the trimethylation of the amino acid lysine, was first discovered in skeletal muscles given its abundance in this tissue ( $95 \%$ of total carnitine in the adults). It plays a central role in the metabolism of fatty acids and maintenance of energy in the body. Carnitine exists in two isomers: D-carnitine and L-carnitine. In animals only L-carnitine is present and D-carnitine inhibits the action of Lcarnitine. Diet intake represents the most abundant source of carnitine in the body, while the remaining $25 \%$ is synthesized in the liver and kidneys from lysine and methionine amino acids (Mitwalli et al, 2005; Silverio et al, 2011).

Skeletal muscles and myocardium are clearly dependent on the action of L-carnitine since fatty acid oxidation is their main source of energy. In cancer-induced cachexia and other chronic illnesses, reduced calorie intake, increased metabolic demands, and pharmacological agents that interfere with L-carnitine absorption in the gut, synthesis or excretion may lead to L-carnitine depletion in these patients (Malaguarnera et al, 2006; Silverio et al, 2011). On this basis, administration of L-carnitine supplements was proposed as a potential therapeutic agent in muscle wasting as improvements in the status of the patients were seen in conditions 
characterized by severe muscle depletion following this treatment (Evangeliou and Vlassopoulos, 2003; Laviano et al, 2006; Silverio et al, 2012; Gramignano et al, 2006).

The biological mechanisms whereby L-carnitine exerts beneficial effects on whole body and muscle weights have been reported in several experimental models of cachexia. As such, a reduction in both tumor-induced triacylglycerol rise and in cytokine levels was demonstrated in septic animals and in rats with sarcoma (Winter et al, 1995). In line with this, several days of treatment with L-carnitine revealed that hepatic lipid metabolism was preserved in cancer cachectic rats (Laviano et al, 2011;Silverio et al, 2011). The upregulation of the enzyme carnitine palmitoyl transferase was also detected in mice bearing colon cancer cells that were treated with L-carnitine, probably as a result of a decrease in levels of proinflammatory cytokines (Liu et al, 2011). Proteasome activity and physical performance (physical activity and mean movement velocity) also improved in rats bearing the Yoshida ascites hepatoma following treatment with L-carnitine for several days (Busquets et al, 2012a). In the present investigation, a significant increase in whole body weight and soleus and gastrocnemius muscle weights was observed in the cancer cachectic rats without inducing any changes in tumor weight, thus suggesting that L-carnitine exerts its effects directly on whole body and muscle metabolism. These findings are consistent with previously reported results conducted on both human studies and animal models (Laviano et al, 2011; Silverio et al, 2011;Busquets et al, 2012a;Gramignano et al, 2006). Moreover, as previously shown (Busquets et al, 2012a) L-carnitine favored food intake in the treated rats, which might have exerted an additional positive effect on the study muscles.

In the current study, muscle fiber type composition and sizes were also explored in two different types of limb muscles: the gastrocnemius and soleus muscles, which are mainly composed by fast- and slow-twitch fibers, respectively. Importantly, the atrophy seen in both types of fibers (type I and type II) of the gastrocnemius was significantly reversed $(21 \%$ and 
331

332

333

$30 \%$ amelioration, respectively) in the cancer cachectic rats treated with L-carnitine, while improvements in fiber sizes were only observed in the slow-twitch fibers of the soleus muscle (16\% amelioration). These are very relevant findings that evidence that the amelioration in muscle fiber sizes underlies the increase in muscle weights detected in the cancer cachectic animals treated with L-carnitine for seven days. In the soleus, however, only a significant increase in the size of the slow-twitch fibers was observed, most likely as a result of the relatively lower degree of atrophy seen in this muscle and the greater proportions of slowtwitch fibers, which are more resistant to protein breakdown (Fermoselle et al, 2012;Lexell et al, 1988;Lexell and Downham, 1992;Lexell, 1995;Puig-Vilanova et al, 2014), contained in this slow-twitch muscle.

Other relevant novel findings in the investigation were the significant decline in the proportions of total muscle abnormalities and internal nuclei counts observed in both gastrocnemius and soleus of the cachectic rats treated with L-carnitine for seven days. These results indicate that in the cachectic muscles, a process of muscle damage and repair has been triggered probably as a response to the presence of the tumors in the rats. These events took place in both slow- and fast-twitch muscle types in a similar fashion, thus implying the predominance of the systemic effects in the cancer cachectic rodents. Similarly, in previous investigations from our group conducted in both patients with cancer-induced cachexia (PuigVilanova et al, 2014) and in experimental models using lung tumor-bearing mice (ChaconCabrera et al, 2014; Chacon-Cabrera et al, 2017;Salazar-Degracia et al, 2016), muscle damage was a characteristic feature in the study muscles. Importantly, L-carnitine induced a significant decline in muscle structural alterations detected in both gastrocnemius and soleus muscles of the tumor-bearing rats. From these results, it would be possibly concluded that Lcarnitine may favor muscle repair and regeneration following injury. These objectives were clearly beyond the scope of the current study but deserve attention in future investigations. 
In the study, levels of total protein ubiquitination and the E3 ligases atrogin-1 and MuRF-1 were significantly reduced in response to treatment with L-carnitine for seven days in the gastrocnemius but not the soleus of the cancer cachectic rats. Levels of autophagy markers, however, did not significantly differ between the study groups of rats in either slowor fast-twitch muscle types. These are relevant findings that may account for the level of improvement of the muscle atrophy seen in both types of muscle fibers in the gastrocnemius of the cancer cachectic rats probably through the attenuation of muscle proteolysis. Moreover, these findings are in agreement with a previous study (Busquets et al, 2012a) from our group in which gene expression of the E3 ligases MuRF-1 and atrogin-1 was also lower in the gastrocnemius of tumor-bearing rats treated with L-carnitine than in the control non-treated animals. On the other hand, no significant changes in protein expression levels of E3 ligases or total protein ubiquitination levels were detected in the soleus muscle of the cachectic rats treated with L-carnitine. The differences in the effects of the pharmacological agent Lcarnitine between slow- and fast-twitch muscle types could be attributed to the reported variability in a muscle-specific manner of cellular processes such as protein synthesis and degradation (Baehr et al, 2017). Likewise, L-carnitine did not elicit any significant effect on protein levels of signaling pathways known to be involved in muscle metabolism and protein breakdown in the soleus of tumor-bearing rats, while a significant decline in FoxO-3 levels was detected in the cachectic gastrocnemius muscle following treatment with L-carnitine. The latter findings suggest that FoxO-3 is likely to be the most relevant signaling pathway driving muscle mass loss and atrophy in the gastrocnemius in this model of cancer-induced cachexia. In line with this, previous studies have also demonstrated the contribution of FoxO-3 to muscle wasting in the lower limb muscles (vastus lateralis, predominantly fast-twitch muscle) of patients with severe muscle atrophy (Fermoselle et al, 2012;Puig-Vilanova et al, 2014).

\section{Conclusions}


381 Treatment with L-carnitine reversed atrophy of slow- and fast-twitch fibers in a muscle382 specific manner in cancer cachectic rats. Muscle morphological features involving muscle 383 damage also ameliorated in response to treatment with L-carnitine of the tumor-bearing rats in 384 both slow- and fast-twitch muscle types in a similar fashion. Expression of proteolytic 385 markers and signaling pathways were attenuated only in the gastrocnemius following L386 carnitine treatment. These data reveal the need to study muscles of different fiber type 387 composition and function to better understand whereby anti-cachectic therapies such as L388 carnitine exert its beneficial effects on skeletal muscles. These findings have potential clinical 389 implications since combinations of various exercise and muscle training modalities with L390 carnitine should be specifically targeted for the muscle groups to be trained. 


\section{2 \\ LIST OF ABBREVIATIONS}

393

394

395

396

397

AKT: serine/threonine kinase 1

ATP: adenosine triphosphate

BSA: bovine serum albumin

CPTA1: carnitine palmitoyl transferase-1

EDTA: ethylenediaminetetraacetic acid

FoxO: forkhead box O

GAPDH: glyceraldehyde 3-phosphate dehydrogenase

HEPES: 4-(2-hydroxyethyl)-1- piperazinnethanesulfonic acid

HRP: horseradish peroxidase

LC3B: microtubule-associated protein 1 light chain 3B

MDA: malondialdehyde

mTOR: mammalian target of rapamycin

MuRF-1: muscle ring finger protein-1

MyHC: myosin heavy chain

$\mathrm{NaCl}$ : Sodium chloride

NaF: Sodium fluoride

NF: nuclear factor

P62: nucleoporin p62

PBST: phosphate buffered saline with teen

PGC: peroxisome proliferator-activated receptor gamma coactivator

PMSF: phenylmethylsulfonyl fluoride

PVDF: polyvinylidene difluoride

SDS: sodium dodecyl sulfate

Sirtuin-1: NAD-dependent protein deacetylase sirtuin-1 


\author{
417 SOD: superoxide dismutase \\ 418 SPSS: Statistical Package for the Social Science
}


420

421

422

423

424

425

426

427

428

429

430

431

432

433

434

435

436

437

438

439

440

441

\section{Ethical publication statement}

We confirm that we have read the Journal's position on issues involved in ethical publication and affirm that this report is consistent with those guidelines

\section{Disclosure of conflict of interest}

None of the authors has any conflict of interest to disclose

\section{Editorial support}

None to declare

\section{AUTHORS CONTRIBUTIONS}

Anna Salazar-Degracia: molecular biology, data analyses and interpretation, results preparation including graphical and tabular representation, and manuscript draft writing

Sílvia Busquets: study design, animal experiments, data analyses and interpretation, and manuscript draft writing

Josep M. Argilès: study design, data analyses and interpretation, results preparation, and manuscript draft writing

Roberto Serpe: study design and data analysis and interpretation

Maria Pérez-Peiró: molecular biology experiments and results preparation

Alba Rojano-Toimil: molecular biology experiments and results preparation

Francisco J. Lopez-Soriano: study design, data analyses and interpretation, results preparation, and manuscript draft writing

Esther Barreiro: study design, data analyses and interpretation, results preparation, and manuscript writing final version

Data will be available upon request to the Authors. 


\section{Literature Cited}

Alvarez FV, Trueba IM, Sanchis JB, Lopez-Rodo LM, Rodriguez Suarez PM, de Cos Escuin JS, Barreiro E, Henar Borrego PM, Vicente CD, Aldeyturriaga JF, Gamez GP, Garrido LP, Leon AP, Izquierdo Elena JM, Novoa Valentin NM, Rivas de Andres JJ, Crespo IR, Velazquez AS, Seijo Maceiras LM, Reina SS, Bujanda DA, Avila Martinez RJ, de Granda Orive JI, Martinez EH, Gude VD, Flor RE, Freixinet Gilart JL, Garcia Jimenez MD, Alarza FH, Sarmiento SH, Honguero Martinez AF, Jimenez Ruiz CA, Sanz IL, Mariscal de AA, Martinez VP, Menal MP, Perez LM, Olmedo Garcia ME, Rombola CA, Arregui IS, Somiedo GM, V, Trivino Ramirez AI, Trujillo Reyes JC, Vallejo C, Lozano PV, Simo GV, Zulueta JJ (2016). Recommendations of the Spanish Society of Pneumology and Thoracic Surgery on the diagnosis and treatment of non-small-cell lung cancer. Arch Bronconeumol 52 Suppl 1:262 .

Baehr LM, West DWD, Marshall AG, Marcotte GR, Baar K, Bodine SC (2017). Musclespecific and age-related changes in protein synthesis and protein degradation in response to hindlimb unloading in rats. J Appl Physiol (1985 ) 122:1336-1350.

Barreiro E (2017). Skeletal Muscle Dysfunction in COPD: Novelties in The Last Decade. Arch Bronconeumol 53:43-44.

Barreiro E, Bustamante V, Cejudo P, Galdiz JB, Gea J, de LP, Martinez-Llorens J, Ortega F, Puente-Maestu L, Roca J, Rodriguez-Gonzalez Moro JM (2015). Guidelines for the evaluation and treatment of muscle dysfunction in patients with chronic obstructive pulmonary disease. Arch Bronconeumol 51:384-395.

Busquets S, Figueras MT, Fuster G, Almendro V, Moore-Carrasco R, Ametller E, Argiles JM, Lopez-Soriano FJ (2004). Anticachectic effects of formoterol: a drug for potential treatment of muscle wasting. Cancer Res 64:6725-6731.

Busquets S, Serpe R, Toledo M, Betancourt A, Marmonti E, Orpi M, Pin F, Capdevila E, Madeddu C, Lopez-Soriano FJ, Mantovani G, Maccio A, Argiles JM (2012a). L-Carnitine: an adequate supplement for a multi-targeted anti-wasting therapy in cancer. Clin Nutr 31:889895.

Busquets S, Toledo M, Marmonti E, Orpi M, Capdevila E, Betancourt A, Lopez-Soriano FJ, Argiles JM (2012b). Formoterol treatment downregulates the myostatin system in skeletal muscle of cachectic tumour-bearing rats. Oncol Lett 3:185-189.

Busquets S, Toledo M, Sirisi S, Orpi M, Serpe R, Coutinho J, Martinez R, Argiles JM, LopezSoriano FJ (2011). Formoterol and cancer muscle wasting in rats: Effects on muscle force and total physical activity. Exp Ther Med 2:731-735.

Carter CS, Cesari M, Ambrosius WT, Hu N, Diz D, Oden S, Sonntag WE, Pahor M (2004). Angiotensin-converting enzyme inhibition, body composition, and physical performance in aged rats. J Gerontol A Biol Sci Med Sci 59:416-423.

Cederholm T, Jensen GL, Correia MITD, Gonzalez MC, Fukushima R, Higashiguchi T, Baptista G, Barazzoni R, Blaauw R, Coats A, Crivelli A, Evans DC, Gramlich L, FuchsTarlovsky V, Keller H, Llido L, Malone A, Mogensen KM, Morley JE, Muscaritoli M, Nyulasi I, Pirlich M, Pisprasert V, de van der Schueren MAE, Siltharm S, Singer P, 
Tappenden K, Velasco N, Waitzberg D, Yamwong P, Yu J, Van GA, Compher C (2019). GLIM criteria for the diagnosis of malnutrition - A consensus report from the global clinical nutrition community. Clin Nutr 38:1-9.

Chacon-Cabrera A, Fermoselle C, Salmela I, Yelamos J, Barreiro E (2015). MicroRNA expression and protein acetylation pattern in respiratory and limb muscles of Parp-1(-/-) and Parp-2(-/-) mice with lung cancer cachexia. Biochim Biophys Acta 1850:2530-2543.

Chacon-Cabrera A, Fermoselle C, Urtreger AJ, Mateu-Jimenez M, Diament MJ, De Kier Joffe ED, Sandri M, Barreiro E (2014). Pharmacological strategies in lung cancer-induced cachexia: effects on muscle proteolysis, autophagy, structure, and weakness. J Cell Physiol 229:1660-1672.

Chacon-Cabrera A, Gea J, Barreiro E (2016a). Short- and Long-Term Hindlimb Immobilization and Reloading: Profile of Epigenetic Events in Gastrocnemius. J Cell Physiol.

Chacon-Cabrera A, Lund-Palau H, Gea J, Barreiro E (2016b). Time-Course of Muscle Mass Loss, Damage, and Proteolysis in Gastrocnemius following Unloading and Reloading: Implications in Chronic Diseases. PLoS One 11:e0164951.

Chacon-Cabrera A, Mateu-Jimenez M, Langohr K, Fermoselle C, Garcia-Arumi E, Andreu AL, Yelamos J, Barreiro E (2017). Role of Parp Activity in Lung Cancer-induced Cachexia: Effects on Muscle Oxidative Stress, Proteolysis, Anabolic Markers and Phenotype. J Cell Physiol.

Evangeliou A, Vlassopoulos D (2003). Carnitine metabolism and deficit--when supplementation is necessary? Curr Pharm Biotechnol 4:211-219.

Fearon K, Strasser F, Anker SD, Bosaeus I, Bruera E, Fainsinger RL, Jatoi A, Loprinzi C, MacDonald N, Mantovani G, Davis M, Muscaritoli M, Ottery F, Radbruch L, Ravasco P, Walsh D, Wilcock A, Kaasa S, Baracos VE (2011). Definition and classification of cancer cachexia: an international consensus. Lancet Oncol 12:489-495.

Fermoselle C, Rabinovich R, Ausin P, Puig-Vilanova E, Coronell C, Sanchez F, Roca J, Gea J, Barreiro E (2012). Does oxidative stress modulate limb muscle atrophy in severe COPD patients? Eur Respir J 40:851-862.

Gonzalez J, de-Torres JP (2017). Lung Cancer and Emphysema. Arch Bronconeumol 53:4748.

Gramignano G, Lusso MR, Madeddu C, Massa E, Serpe R, Deiana L, Lamonica G, Dessi M, Spiga C, Astara G, Maccio A, Mantovani G (2006). Efficacy of 1-carnitine administration on fatigue, nutritional status, oxidative stress, and related quality of life in 12 advanced cancer patients undergoing anticancer therapy. Nutrition 22:136-145.

Izquierdo Alonso JL (2016). Comorbidities in chronic obstructive pulmonary diasease. Arch Bronconeumol 52:547-548.

Kraft M, Kraft K, Gartner S, Mayerle J, Simon P, Weber E, Schutte K, Stieler J, Koula-Jenik H, Holzhauer P, Grober U, Engel G, Muller C, Feng YS, Aghdassi A, Nitsche C, Malfertheiner P, Patrzyk M, Kohlmann T, Lerch MM (2012). L-Carnitine-supplementation in advanced pancreatic cancer (CARPAN)--a randomized multicentre trial. Nutr J 11:52. 
Laviano A, Meguid MM, Guijarro A, Muscaritoli M, Cascino A, Preziosa I, Molfino A, Rossi FF (2006). Antimyopathic effects of carnitine and nicotine. Curr Opin Clin Nutr Metab Care 9:442-448.

Laviano A, Seelaender M, Sanchez-Lara K, Gioulbasanis I, Molfino A, Rossi FF (2011). Beyond anorexia -cachexia. Nutrition and modulation of cancer patients' metabolism: supplementary, complementary or alternative anti-neoplastic therapy? Eur J Pharmacol 668 Suppl 1:S87-S90.

Lexell J (1995). Human aging, muscle mass, and fiber type composition. J Gerontol A Biol Sci Med Sci 50 Spec No:11-16.

Lexell J, Downham D (1992). What is the effect of ageing on type 2 muscle fibres? J Neurol Sci 107:250-251.

Lexell J, Taylor CC, Sjostrom M (1988). What is the cause of the ageing atrophy? Total number, size and proportion of different fiber types studied in whole vastus lateralis muscle from 15- to 83-year-old men. J Neurol Sci 84:275-294.

Liu S, Wu HJ, Zhang ZQ, Chen Q, Liu B, Wu JP, Zhu L (2011). L-carnitine ameliorates cancer cachexia in mice by regulating the expression and activity of carnitine palmityl transferase. Cancer Biol Ther 12:125-130.

Lopez-Soriano J, Argiles JM, Lopez-Soriano FJ (1997). Sequential changes in lipoprotein lipase activity and lipaemia induced by the Yoshida AH-130 ascites hepatoma in rats. Cancer Lett 116:159-165.

Malaguarnera M, Risino C, Gargante MP, Oreste G, Barone G, Tomasello AV, Costanzo M, Cannizzaro MA (2006). Decrease of serum carnitine levels in patients with or without gastrointestinal cancer cachexia. World J Gastroenterol 12:4541-4545.

Mitwalli AH, Al-Wakeel JS, Alam A, Tarif N, Abu-Aisha H, Rashed M, Al NN (2005). Lcarnitine supplementation in hemodialysis patients. Saudi J Kidney Dis Transpl 16:17-22.

Puig-Vilanova E, Martinez-Llorens J, Ausin P, Roca J, Gea J, Barreiro E (2015). Quadriceps muscle weakness and atrophy are associated with a differential epigenetic profile in advanced COPD. Clin Sci (Lond) 128:905-921.

Puig-Vilanova E, Rodriguez DA, Lloreta J, Ausin P, Pascual-Guardia S, Broquetas J, Roca J, Gea J, Barreiro E (2014). Oxidative stress, redox signaling pathways, and autophagy in cachectic muscles of male patients with advanced COPD and lung cancer. Free Radic Biol Med 79C:91-108.

Salazar-Degracia A, Blanco D, Vila-Ubach M, de BG, de Solorzano CO, Montuenga LM, Barreiro E (2016). Phenotypic and metabolic features of mouse diaphragm and gastrocnemius muscles in chronic lung carcinogenesis: influence of underlying emphysema. J Transl Med $14: 244$.

Salazar-Degracia A, Busquets S, Argiles JM, Lopez-Soriano FJ, Barreiro E (2017). Formoterol attenuates increased oxidative stress and myosin protein loss in respiratory and limb muscles of cancer cachectic rats. PeerJ 5:e4109. 
564 Silverio R, Laviano A, Rossi FF, Seelaender M (2011). 1-carnitine and cancer cachexia:

565 Clinical and experimental aspects. J Cachexia Sarcopenia Muscle 2:37-44.

566 Silverio R, Laviano A, Rossi FF, Seelaender M (2012). L-Carnitine induces recovery of liver 567 lipid metabolism in cancer cachexia. Amino Acids 42:1783-1792.

568 Szefel J, Kruszewski WJ, Ciesielski M, Szajewski M, Kawecki K, Aleksandrowicz-Wrona E, 569 Jankun J, Lysiak-Szydlowska W (2012). L-carnitine and cancer cachexia. I. L-carnitine 570 distribution and metabolic disorders in cancer cachexia. Oncol Rep 28:319-323.

571 Toledo M, Busquets S, Penna F, Zhou X, Marmonti E, Betancourt A, Massa D, Lopez572 Soriano FJ, Han HQ, Argiles JM (2016). Complete reversal of muscle wasting in 573 experimental cancer cachexia: Additive effects of activin type II receptor inhibition and beta5742 agonist. Int J Cancer 138:2021-2029.

Toledo M, Busquets S, Sirisi S, Serpe R, Orpi M, Coutinho J, Martinez R, Lopez-Soriano FJ, 576 Argiles JM (2011). Cancer cachexia: physical activity and muscle force in tumour-bearing 577 rats. Oncol Rep 25:189-193.

Toledo M, Springer J, Busquets S, Tschirner A, Lopez-Soriano FJ, Anker SD, Argiles JM (2014). Formoterol in the treatment of experimental cancer cachexia: effects on heart 580 function. J Cachexia Sarcopenia Muscle 5:315-320.

von HS, Anker SD (2014). Treatment of cachexia: an overview of recent developments. J Am 582 Med Dir Assoc 15:866-872.

Winter BK, Fiskum G, Gallo LL (1995). Effects of L-carnitine on serum triglyceride and 


\section{FIGURE LEGENDS}

Figure 1: (A) Representative immunoblots and (B) mean values and standard deviation of the carnitine palmitoyl transferase-1 in the gastrocnemius and soleus muscles. GAPDH protein bands are shown as the loading control for all the immunoblots. Protein levels measured by optical densities expressed in arbitrary units (O.D, a.u.). Definition of abbreviations: CPT1A, carnitine palmitoyl transferase-1. Statistical significance: ${ }^{*} \mathrm{p} \leq 0.05$ between cancer-cachexia and cancer-cachexia+carnitine animals.

Figure 2: Representative examples of muscle fibers (x200) within gastrocnemius (upper panels) and soleus (bottom panels) muscles of cancer-cachexia (left panels) and cancercachexia+carnitine rats (right panels). Fast-twitch fibers were positively stained with the corresponding antibody (brown color), while the non-stained fibers were the slow-twitch fibers (all panels).

Figure 3: Representative examples of muscle morphology in the (A) gastrocnemius and (B) soleus muscles of cancer-cachexia and cancer-cachexia+carnitine groups of animals. Black arrows point towards internal nuclei. Mean values and standard deviation of muscle morphology (total abnormalities, internal nuclei, and cellular inflammation proportions, see methods for details) within (C) gastrocnemius and (D) soleus muscles of cancer-cachexia and cancer-cachexia+carnitine rats. Statistical significance: $* * \mathrm{p} \leq 0.01$ and $* * * \mathrm{p} \leq 0.001$ between cancer-cachexia and cancer-cachexia+carnitine animals.

Figure 4: (A) Representative immunoblots and (B) mean values and standard deviation of MDA-protein adducts, SOD1, SOD2, and catalase, in the gastrocnemius and (C) representative immunoblots and (D) mean values and standard deviation in soleus muscles. GAPDH protein bands are shown as the loading control for all the immunoblots. Protein levels measured by optical densities expressed in arbitrary units (O.D, a.u.). Definition of 
612 abbreviations: MDA, malondialdehyde; SOD, superoxide dismutase. Statistical significance:

$613 * \mathrm{p} \leq 0.05$ between cancer-cachexia and cancer-cachexia+carnitine animals.

614 Figure 5: (A) Representative immunoblots and (B) mean values and standard deviation of 615 Sirtuin-1, PGC-1 alpha, NF-kB p65 and FoxO-3 in the gastrocnemius and (C) representative 616 immunoblots and (D) mean values and standard deviation in soleus muscles. GAPDH protein 617 bands are shown as the loading control for all the immunoblots. Protein levels measured by 618 optical densities expressed in arbitrary units (O.D, a.u.). Definition of abbreviations: PGC619 1alpha, peroxisome proliferator-activated receptor gamma coactivator 1-alpha; NF-kB p65, 620 nuclear factor kappa-light-chain-enhancer of activated $\mathrm{B}$ cells p65; FoxO-3, forkhead box 621 protein O3. Statistical significance: * $\mathrm{p} \leq 0.05$ between cancer-cachexia and cancer622 cachexia+carnitine animals.

Figure 6: (A) Representative immunoblots and (B) mean values and standard deviation of AKT and mTOR, in the gastrocnemius and (C) representative immunoblots and (D) mean values and standard deviation in soleus muscles. GAPDH protein bands are shown as the loading control for all the immunoblots. Protein levels measured by optical densities expressed in arbitrary units (O.D, a.u.). Definition of abbreviations: AKT, serine/threonine kinase 1; mTOR, mammalian target of rapamycin.

Figure 7: (A) Representative immunoblots and (B) mean values and standard deviation of the proteolytic markers total ubiquitinated proteins, MuRF-1, and atrogin-1, in the gastrocnemius and (C) representative immunoblots and (D) mean values and standard deviation in soleus muscles. GAPDH protein bands are shown as the loading control for all the immunoblots. Protein levels measured by optical densities expressed in arbitrary units (O.D, a.u.). Definition of abbreviations: MuRF-1, muscle ring finger protein-1. Statistical significance: ${ }^{*} \mathrm{p} \leq 0.05$ and $* * p \leq 0.01$ between cancer-cachexia and cancer-cachexia+carnitine animals. 
636 Figure 8: (A) Representative immunoblots and (B) mean values and standard deviation of the 637 p62 and $\mathrm{LC} 3 \mathrm{~B}$, in the gastrocnemius and (C) representative immunoblots and (D) mean 638 values and standard deviation in soleus muscles. GAPDH protein bands are shown as the 639 loading control for all the immunoblots. Protein levels measured by optical densities 640 expressed in arbitrary units (O.D, a.u.). Definition of abbreviations: p62, nucleoporin p62; $641 L C 3 B$, microtubule-associated protein 1 light chain 3B. 
Table 1. Physiological characteristics of the study groups of animals

\begin{tabular}{lll} 
& Cancer-cachexia & Cancer-cachexia+carnitine \\
\hline Food intake (g/100g IBW) & $57.2(6.6)$ & $68.3(6.3)^{*}$ \\
Tumor weight (g) & $36.0(4.3)$ & $34.8(7.3)$ \\
Initial body weight (g) & $127.1(8.2)$ & $120.5(5.8)$ \\
Final body weight (g),without tumor & $128.8(7.5)$ & $133.3(9.9)$ \\
Body weight gain (\%) & $+1.7(9.1)$ & $+10.6(6.7)^{*}$ \\
Gastrocnemius weight (mg/100g IBW) & $525.7(23.0)$ & $569.7(34.8)^{* *}$ \\
Soleus weight (mg/100g IBW) & $34.8(2.6)$ & $40.2(3.3)^{* *}$ \\
\hline
\end{tabular}

Variables are represented as mean (standard deviation). Food intake and muscle weight are expressed in $\mathrm{g}$ and $\mathrm{mg} / 100 \mathrm{~g}$ of IBW, respectively. Definition of abbreviations: $\mathrm{g}$, gram; mg, milligram; IBW, initial body weight. Statistical significance: $* p \leq 0.05$ and $* * p \leq 0.01$ between cancer-cachexia rats and cancer-cachexia rats treated with Lcarnitine. 
Table 2. Muscle fiber type composition and morphometry in all study groups

\begin{tabular}{lll} 
& Cancer-cachexia & Cancer-cachexia+carnitine \\
\hline Gastrocnemius & & \\
Type I fibers $(\%)$ & $23.6(5.0)$ & $21.0(4.8)$ \\
Type II fibers $(\%)$ & $76.4(5.0)$ & $79.0(4.8)$ \\
Type I fibers areas $\left(\mu \mathrm{m}^{2}\right)$ & $434.5(62.6)$ & $524.9(81.4)^{*}$ \\
Type II fibers areas $\left(\mu \mathrm{m}^{2}\right)$ & $524.9(74.4)$ & $684.6(35.9)^{* * *}$ \\
Soleus & & $70.7(7.6)$ \\
Type I fibers $(\%)$ & $65.2(2.0)$ & $29.3(7.6)$ \\
Type II fibers $(\%)$ & $34.8(2.0)$ & $967.0(71.7)^{* *}$ \\
Type I fibers areas $\left(\mu \mathrm{m}^{2}\right)$ & $835.1(71.8)$ & $754.5(44.7)$ \\
Type II fibers areas $\left(\mu \mathrm{m}^{2}\right)$ & $733.3(63.5)$ & \\
\hline
\end{tabular}

Variables are represented as mean (standard deviation). Definition of abbreviations: $\mu \mathrm{m}$, micrometer. Statistical significance: ${ }^{*} p \leq 0.05$, ** $p \leq 0.01$ and $* * * p \leq 0.001$ between cancercachexia rats and cancer-cachexia rats treated with L-carnitine. 
Figure 1. Salazar-Degracia et al.

A

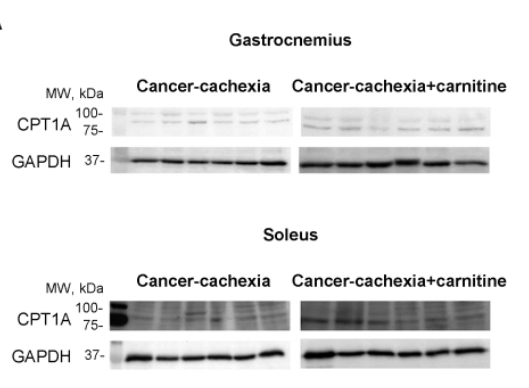

B

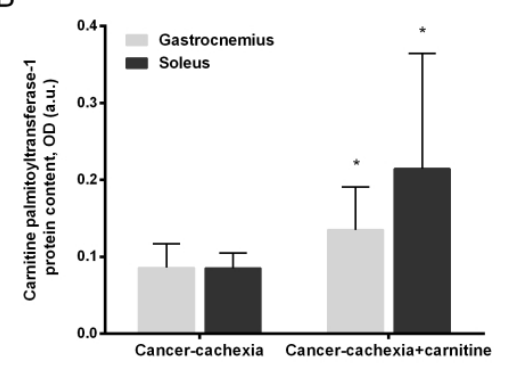

Figure 1

$298 \times 211 \mathrm{~mm}(300 \times 300$ DPI)

John Wiley \& Sons, Inc. 
Figure 2.Salazar-Degracia et al.
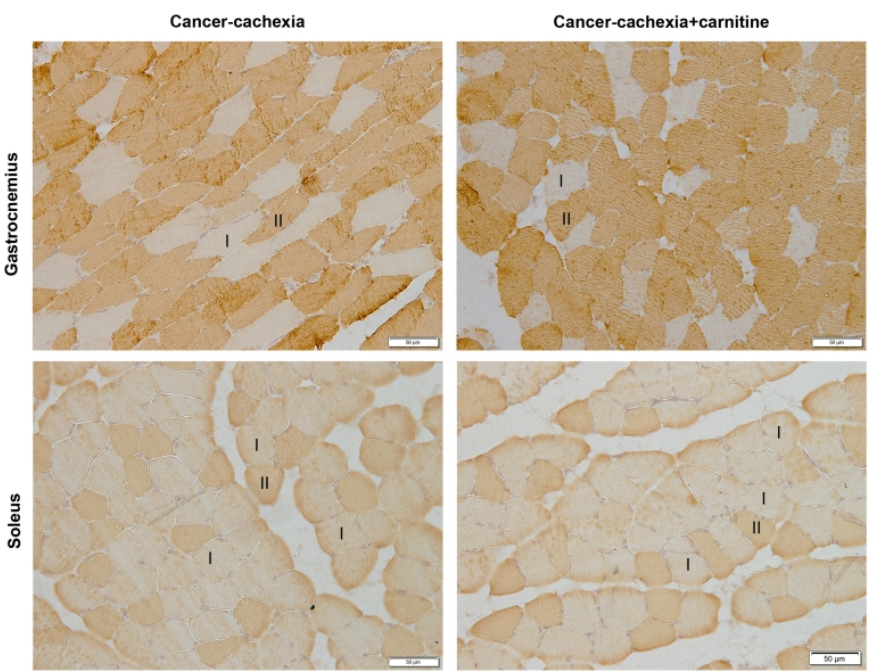

Fgure 2

John Wiley \& Sons, Inc. 


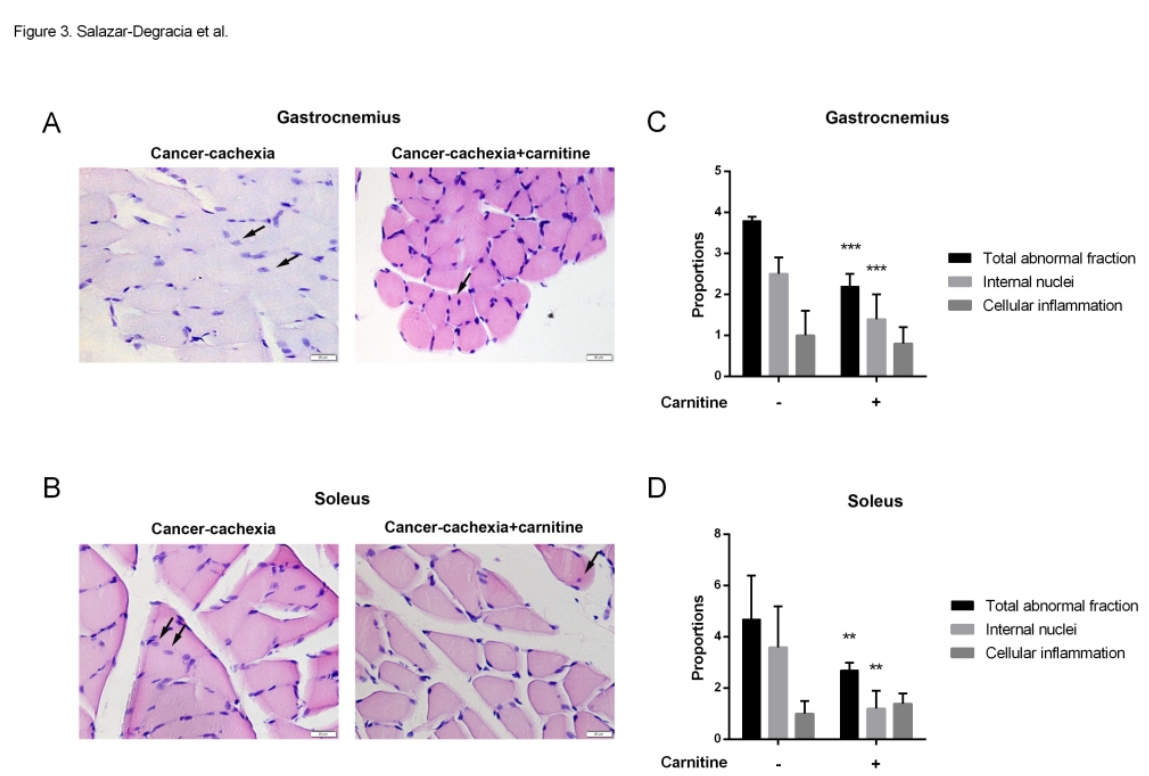

Figure 3

John Wiley \& Sons, Inc. 


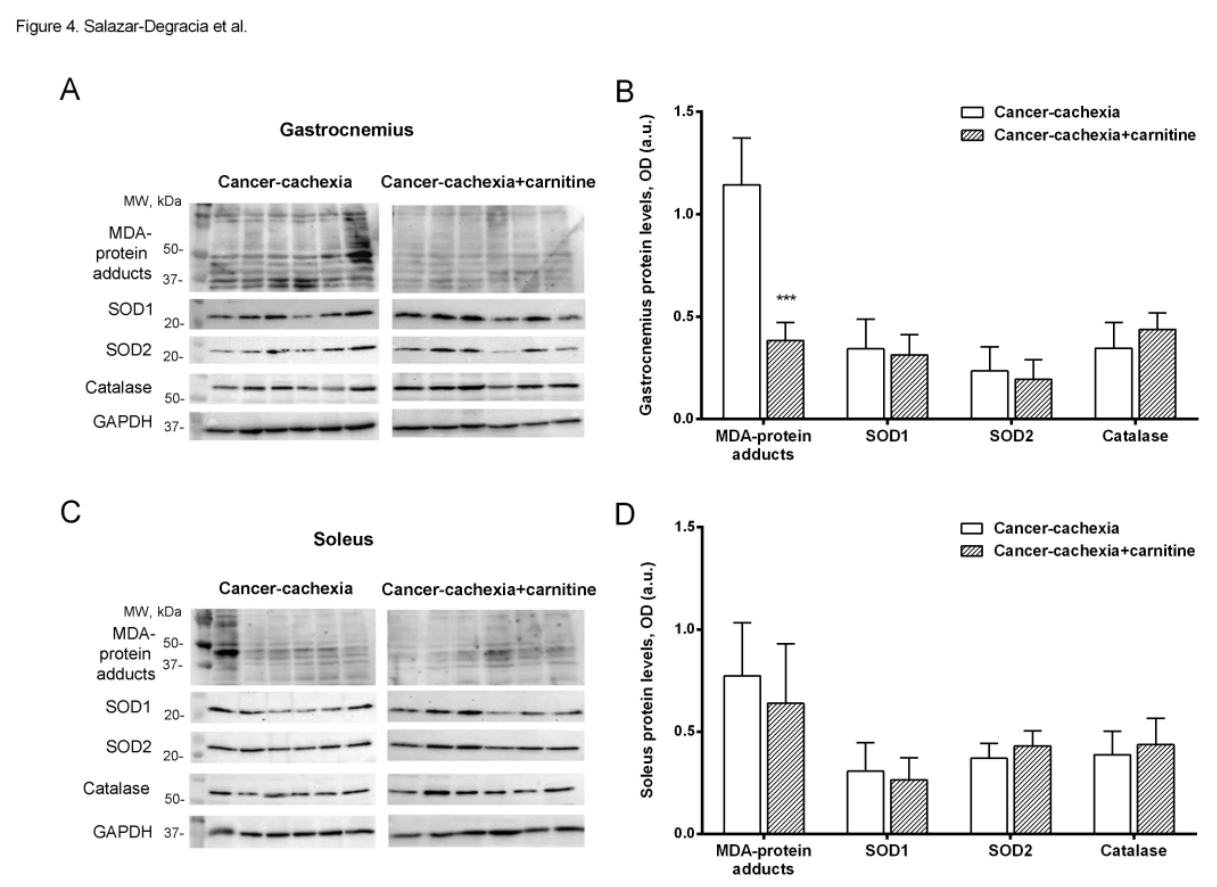

Figure 4

John Wiley \& Sons, Inc. 
A
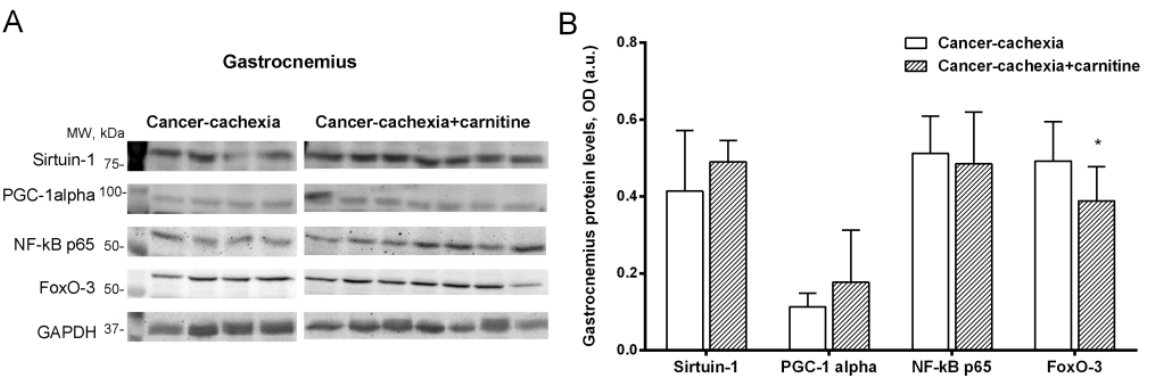

C
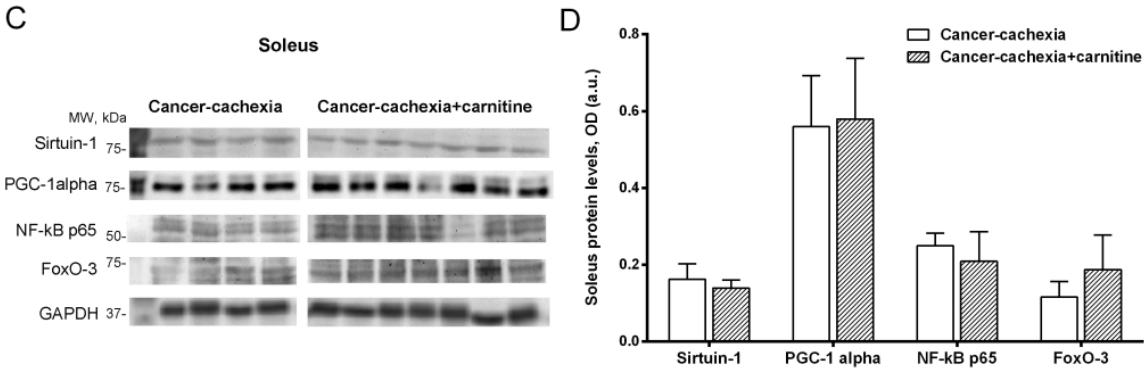

Figure 5 
Figure 6. Salazar-Degracia et al.

A

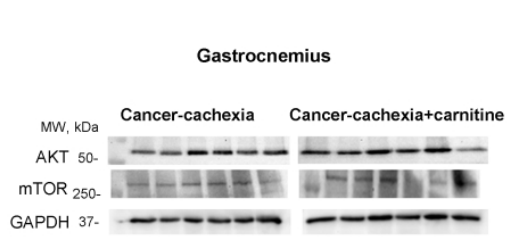

C

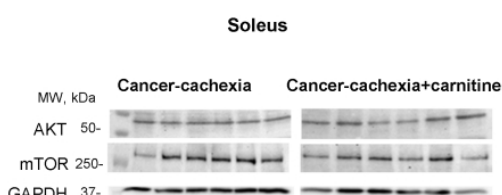

B

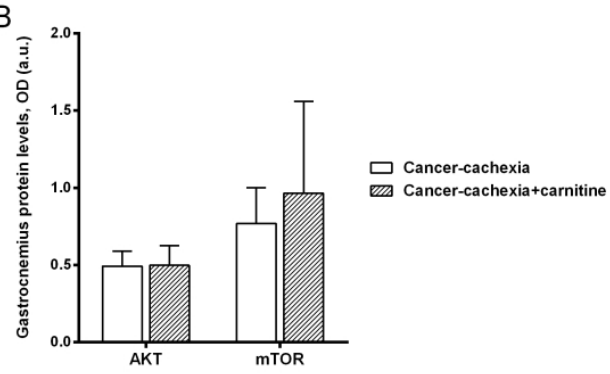

D

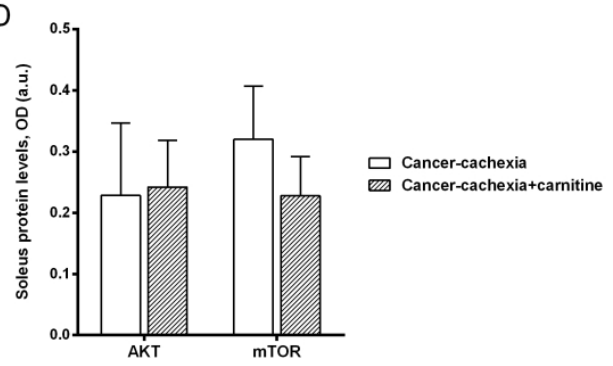

Figure 6

John Wiley \& Sons, Inc. 
Figure 7. Salazar-Degracia et al.

A

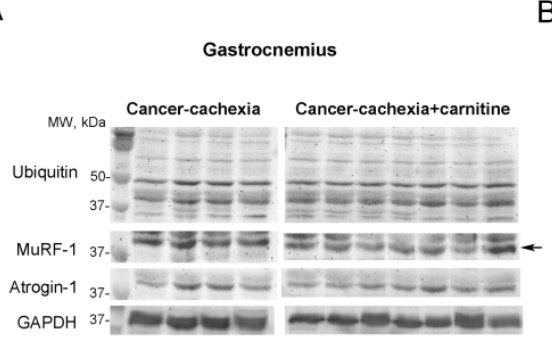

C

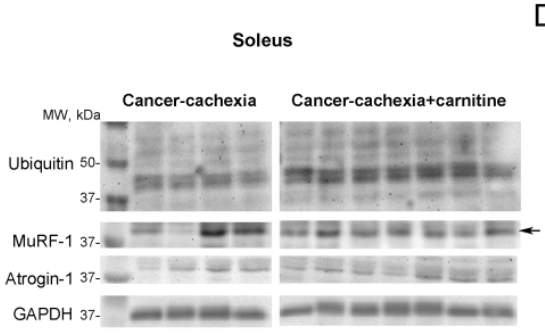

B

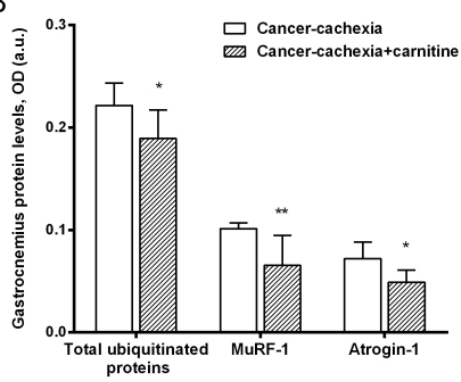

D $\quad \begin{array}{ll}1.0 \\ \square\end{array}$

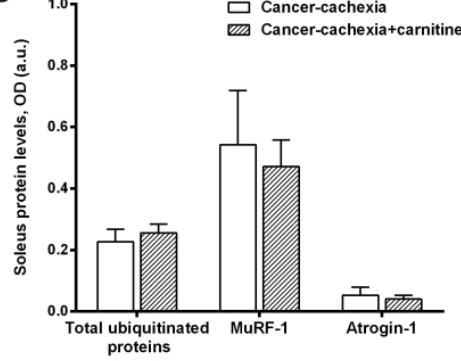

Figure 7

John Wiley \& Sons, Inc. 


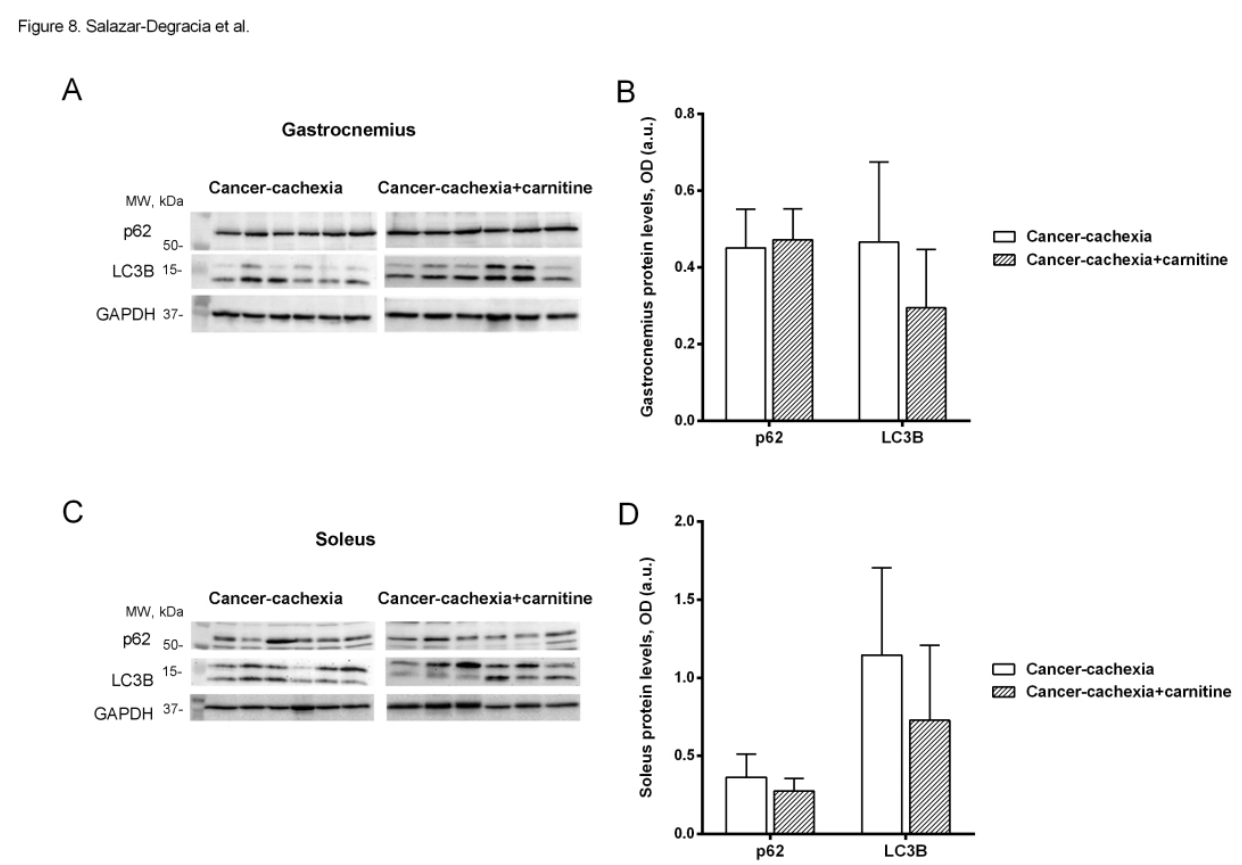

Figure 8

John Wiley \& Sons, Inc. 